\title{
WHAT IS THE BEST ANGULAR DENSITY OF MULTIPATH IN MIMO CHANNELS?
}

\author{
G. Levin and S. Loyka \\ School of Information Technology and Engineering \\ University of Ottawa \\ 161 Louis Pasteur, Ottawa, Ontario, Canada, K1N 6N5 \\ Tel: (613) 562-5800 ext. 2198, Fax: (613) 562-5175 \\ Email: \{glevin, sloyka\}@site.uottawa.ca
}

\begin{abstract}
While various models of multipath angular density are used for modeling MIMO fading channels, it is not known which one is the best, i.e. gives the highest channel capacity. We answer this question in two steps. First, we show that the asymptotic capacity of a broad class of MIMO channels (not necessarily Rayleigh-fading) with an arbitrary correlation structure does not depend on particular channel distribution, but only on the correlation between antennas. Second, the best multipath angular density, which eliminates the correlation and thus maximizes the asymptotic capacity of uniform linear antenna arrays, is shown to be non-uniform, which implies that the popular Clarke's model does not represent the best-case scenario. We identify the best angular density for different values of the antenna spacing. The Gaussian angular density (adopted in IEEE 802.11n) is shown to be near optimum when the spacing equals to the wavelength. Based on these results, practical guidelines for the antenna array design are given.
\end{abstract}

\section{INTRODUCTION}

There are two major propagation-related factors that affect the capacity of MIMO fading channels: antenna array configuration (e.g. element spacing) and the angular density of incoming multipath. A number of capacity optimization problems, which take into account these factors, have been formulated and solved. A systematic framework for adapting the antenna spacing to maximize the channel capacity in a sparse multipath environment is developed in [1]. The effect of capacity saturation in circular antenna arrays of a fixed aperture with the uniform angular density of the multipath has been analyzed in [2]. The uniform density within a sector has been investigated and the requirement on antenna element spacing has been formulated in [3]. Various models of nonuniform angular densities (e.g. Gaussian, Laplacian, cosine etc.) have been proposed and validated under various propagation conditions [4].

The question traditionally asked in the prior works, in one form or another, is "what is the effect of such-and-such angular density on the channel capacity (correlation, diversity, etc.)?". Here, we turn the problem around and ask the following question: "what is the best possible angular density of multipath?". We are not targeting in building a model for a specific propagation environment, but rather we wish to know what is the best possible environment, in terms of multipath angular density. While being an intriguing question from a theoretical viewpoint, it is also practically-relevant. Specifically, such best density, once found, can serve as a benchmark for practically-existing densities to compare with, i.e. "how far away is such-and-such density (in such-and-such propagation conditions) from the best possible one?", which also can be transformed into a similar question in terms of the channel capacity (bit error rate etc.).

In the present paper, this problem is solved for a broad class of MIMO channels with uniform linear antenna arrays (ULA). While the relationship between propagation channel characteristics, antenna array design and the channel capacity are rarely amenable to closed-form analysis, we go around this problem by using an asymptotic approximation of the channel capacity (when the number of antennas is large), which allows us to obtain the best angular density in a closed-form and for a general class of fading channels (not only Rayleigh), and also an insight as to why it is of that particular form. This sizeasymptotic approach has already been used successfully in a number of studies, and has also been proven to be reasonably accurate for channels with a moderate number of antennas [5].

We follow a standard approach in the antenna array literature and adopt the far-field assumption, as well as we use a simplified model of isotropic (ideal) antenna elements with no mutual coupling. The main contributions are as follows:

- We show that when the number of antennas is large, the asymptotic outage capacity of a broad class of MIMO channels (not necessarily Rayleigh-fading) with an arbitrary correlation structure (not necessarily unitary-independent-unitary UIU, as in [5]) does not depend on a particular channel distribution, but only on the correlation between antennas. The following widely-used models are included as special cases: the classic i.i.d. and correlated Rayleigh-fading channel with separable (Kronecker) correlation structure [4], and i.i.d. zero-mean (not necessarily Rayleigh-fading) channel with finite forth-order statistics.

- Using Szego Theorem [6], we characterize the best multipath angular density that eliminates the correlation between all antennas and thus maximizes the asymptotic capacity of the broad class of MIMO channels, when the receive ULA and the multipath are located on a plane (2-D case). Specifically, (i) When the antenna spacing is greater than half a wavelength, there are infinite number of such densities (a condition on a density to belong to that class is given); (ii) When the spacing equals to one wavelength, the Gaussian angular density (adopted in IEEE 802.11n as a standard model [4]) with the peak at the array broadside direction is near optimum; (iii) When the antenna spacing is half a wavelength, there is a single best density (given in a closed form); finally, (iv) When the spacing is less than the wavelength, there exists no density that is able to eliminate correlation between all antennas and, hence, to achieve the maximum asymptotic capacity (equal to that of an uncorrelated 
channel). The capacity-maximizing density is shown to be non-uniform, from which it follows that the popular Clarke's (Jakes's) model (e.g. the uniform angular density) does not represent the best case propagation scenario.

\section{ASYMPTOTIC CAPACITY OF MIMO CHANNELS}

Consider a MIMO channel with $n_{t}$ transmit and $n_{r}$ receive antennas under the following assumptions: (i) the channel state information (CSI) is available at the receive ( $\mathrm{Rx})$ end but not at the transmit (Tx) end, (ii) the total transmitted power is constrained to $P_{T}$ and does not depend on $n_{t}$, (iii) the noise at the $\mathrm{Rx}$ end is spatially uncorrelated circular symmetric Gaussian and has identical power per each Rx antenna. The instantaneous capacity (i.e. the capacity of a given channel realization) per $\mathrm{Rx}$ antenna of a frequency flat quasi-static MIMO channel in natural units $[n a t]$ is given by

$$
C=n_{r}^{-1} \ln \operatorname{det}\left[\mathbf{I}+\gamma_{0} / n_{t} \cdot \mathbf{H} \mathbf{H}^{H}\right],
$$

where $\mathbf{H}$ is the channel matrix whose elements $H_{k m}$, $k=1 \ldots n_{r}, m=1 \ldots n_{t}$ represent a complex gain from the $m^{\text {th }}$ transmit to the $k^{\text {th }}$ receive antenna, $\mathbf{H}^{H}$ is the Hermitian conjugate of $\mathbf{H}$, and $\gamma_{0}$ is the SNR per Rx antenna. Without loss of generality $\mathbf{H}$ is normalized so that $E\|\mathbf{H}\|^{2}=n_{t} n_{r}$, where \|\| is the Frobenius norm. The following notations are used below: (i) $\boldsymbol{\Gamma}_{1}=E\left\{\operatorname{vec}(\mathbf{H}) \cdot \operatorname{vec}^{H}(\mathbf{H})\right\}$, $\boldsymbol{\Gamma}_{2}=E\left\{\operatorname{vec}\left(\mathbf{H}^{H}\right) \cdot \operatorname{vec}^{H}\left(\mathbf{H}^{H}\right)\right\} . \quad$ (ii) $\quad \mathbf{R}_{r}=n_{t}^{-1} E\left\{\mathbf{H} \mathbf{H}^{H}\right\}$, $\mathbf{R}_{t}=n_{r}^{-1} E\left\{\mathbf{H}^{H} \mathbf{H}\right\}$. Note that due to the adopted normalization, $\operatorname{tr} \mathbf{R}_{r}=n_{r}$ and $t r \mathbf{R}_{t}=n_{t}$, where $t r$ stands for trace.

Definition 1: The total fourth order cumulant of $\mathbf{H}$ is defined as

$$
K_{4}(\mathbf{H})=\sum_{k, m=1}^{n_{t}} \sum_{n, l=1}^{n_{r}} \kappa_{4}\left(H_{n m} H_{n k} H_{l k} H_{l m}\right),
$$

where $\kappa_{4}\left(H_{n m} H_{n k} H_{l k} H_{l m}\right)$ is the fourth order cumulant of circular symmetric random variables.

Definition 2: The pseudo-norm of $\boldsymbol{\Gamma}_{1}, \boldsymbol{\Gamma}_{2}$ :

$$
\left\|\boldsymbol{\Gamma}_{1}\right\|_{Q}=\left(\sum_{k, l=1}^{n_{t}}\left|\operatorname{tr} \mathbf{Q}_{k l}\right|^{2}\right)^{1 / 2},\left\|\boldsymbol{\Gamma}_{2}\right\|_{Q}=\left(\sum_{k, l=1}^{n_{r}}\left|\operatorname{tr} \mathbf{G}_{k l}\right|^{2}\right)^{1 / 2},
$$

where $\mathbf{Q}_{k l}=E\left\{\mathbf{h}_{k} \mathbf{h}_{l}^{H}\right\}$ and $\mathbf{G}_{k l}=E\left\{\mathbf{g}_{k} \mathbf{g}_{l}^{H}\right\}$ are the blocks of $\boldsymbol{\Gamma}_{1}$ and $\boldsymbol{\Gamma}_{2}, \mathbf{h}_{k}$ and $\mathbf{g}_{k}$ are the $k^{\text {th }}$ columns of $\mathbf{H}$ and $\mathbf{H}^{H}$ respectively.

In general, \|\|$_{Q}$ is not a norm in the usual sense, since it is possible that for two matrices $\boldsymbol{\Gamma}_{1} \neq \boldsymbol{\Gamma}_{2},\left\|\boldsymbol{\Gamma}_{1}-\boldsymbol{\Gamma}_{2}\right\|_{Q}=0$. However, as we show later, in some cases the pseudo-norm is equivalent to the Frobenius norm.

Theorem 1: Let $\mathbf{H}$ be a complex circular symmetric random matrix (not necessarily Gaussian), and the following conditions are satisfied:

$$
\text { (i) } \begin{gathered}
\lim _{n_{t}, n_{r} \rightarrow \infty}\left[n_{t}^{-2}\left(\left\|\boldsymbol{\Gamma}_{1}\right\|_{Q}^{2}+\left\|\boldsymbol{\Gamma}_{2}\right\|_{Q}^{2}\right)-\left\|\mathbf{R}_{r}\right\|^{2}\right]=0^{1} \\
\text { (ii) } \lim _{n_{t}, n_{r} \rightarrow \infty} n_{t}^{-2} K_{4}(\mathbf{H})=0
\end{gathered}
$$

\footnotetext{
${ }^{1}$ We show later on that this condition implies that $n_{t}$ increases much faster than $n_{r}$.
}

Then, as $n_{t}$ or both $n_{t}$ and $n_{r}$ go to infinity, the instantaneous capacity per $\mathrm{Rx}$ antenna is

$$
C \stackrel{p}{\rightarrow} n_{r}^{-1} \ln \operatorname{det}\left[\mathbf{I}+\gamma_{0} \mathbf{R}_{r}\right],
$$

where $\stackrel{p}{\rightarrow}$ denotes convergence in probability.

Proof: due to Slutsky Theorem, and the fact that under conditions (4) (5), for any $\varepsilon>0, \operatorname{Pr}\left\{\left\|\mathbf{H H}^{H} / n_{t}-\mathbf{R}_{r}\right\| \geq \varepsilon\right\} \rightarrow 0$ as $n_{t}$ or both $n_{t}$ and $n_{r}$ go to infinity, where $\operatorname{Pr}\{\}$ denotes probability.

Theorem 1 allows splitting the effect of correlation at the transmit and receive ends and indicates that in asymptotic approximation the channel capacity does not depend on a particular distribution of $\mathbf{H}$, nor on the channel correlation structure, but only on $\mathbf{R}_{r}$. Moreover, under the conditions of Theorem 1, the channel "hardens" as the number of antennas increases, i.e. $C$ converges to a deterministic value equal to the upper bound. As a special case, Theorem 1 includes a number of popular channel models for which result in (6) is known:

(i) $\mathbf{H}$ is i.i.d. complex circular symmetric. Based on Definition 1, it is straightforward to show that in this case $K_{4}(\mathbf{H})=n_{t} n_{r}\left(E\left|H_{k m}\right|^{4}-2\right)$. Thus, if $\mathbf{H}$ is i.i.d. complex circular symmetric Gaussian $\left(E\left|H_{k m}\right|^{4}=2\right)$, then $K_{4}(\mathbf{H}) \equiv 0$, so that (5) always holds true. If $\mathbf{H}$ is not Gaussian, but $E\left|H_{k m}\right|^{4}$ is finite, it is straightforward to show that $n_{t}^{-2} K_{4}(\mathbf{H})$ behaves as $n_{r} / n_{t}$, and $n_{t}^{-2}\left(\left\|\boldsymbol{\Gamma}_{r}\right\|_{Q}^{2}+\left\|\boldsymbol{\Gamma}_{t}\right\|_{Q}^{2}\right)-\left\|\mathbf{R}_{r}\right\|^{2}=n_{r} / \sqrt{n_{t}}$, i.e. Theorem 1 holds if $\lim _{n_{t}, n_{t} \rightarrow \infty} n_{r} / \sqrt{n_{t}}=0$, or equivalently $n_{t}$ has to increase much faster than $n_{r}$.

(ii) The channel correlation structure is separable and follows Kronecker correlation model [4], i.e. $\boldsymbol{\Gamma}_{1}=\mathbf{R}_{t}^{*} \otimes \mathbf{R}_{r}$, $\boldsymbol{\Gamma}_{2}=\mathbf{R}_{r}^{*} \otimes \mathbf{R}_{t}$, where $\otimes$ denotes the Kronecker product of two matrices, and $\mathbf{R}_{t}^{*}$ is the complex conjugate of $\mathbf{R}_{t}$ elementwise. It is straightforward to show, based on Definition 2 , that in this case $\left\|\boldsymbol{\Gamma}_{1}\right\|_{Q}^{2}=n_{r}^{2}\left\|\mathbf{R}_{t}\right\|^{2},\left\|\boldsymbol{\Gamma}_{2}\right\|_{Q}^{2}=n_{t}^{2}\left\|\mathbf{R}_{r}\right\|^{2}$ (the pseudo-norm and the Frobenius norm are equivalent), and (4) becomes thereby

$$
\lim _{n_{t}, n_{r} \rightarrow \infty}\left[n_{t}^{-2}\left(\left\|\boldsymbol{\Gamma}_{1}\right\|_{Q}^{2}+\left\|\boldsymbol{\Gamma}_{2}\right\|_{Q}^{2}\right)-\left\|\mathbf{R}_{r}\right\|^{2}\right]=\lim _{n_{t}, n_{r} \rightarrow \infty}\left(\frac{n_{r}}{n_{t}}\left\|\mathbf{R}_{t}\right\|\right)^{2}=0,(7)
$$

Consider a Rayleigh-fading and a non Rayleigh-fading MIMO channels (both with the same $\mathbf{R}_{r}$ ) such that conditions (4) and (5) are satisfied. From Theorem 1, when the number of antennas is large in both channels, their instantaneous capacity converges to the same value given by the RHS of (6). This motivates the following definition:

Definition 3: A MIMO channel is said to be asymptotically Rayleigh-like (in terms of capacity) if it satisfies conditions (4) and (5).

Note that uncorrelated keyhole channels are not Rayleighlike, since in this case (5) is not satisfied as $\lim _{n_{t} \rightarrow \infty} n_{t}^{-2} K_{4}(\mathbf{H})=n_{r}^{2} \neq 0$.

Corollary 1: Consider a MIMO channel that satisfies the conditions of Theorem 1 and whose correlation matrix $\mathbf{R}_{r}$ has 
a Toeplitz structure ${ }^{2}$, i.e. $R_{n m}=R_{n-m}, n, m=1 \ldots n_{r}$, where $R_{n m}$ is an element of $\mathbf{R}_{r}$, and $R_{n-m}$ is the matrix-generating vector. Assume that $\mathbf{R}_{r}$ is non-degenerate and squaresummable, i.e. $0<\lim _{n_{r} \rightarrow \infty} \sum_{n=-n_{r}+1}^{n_{r}-1}\left|R_{n}\right|^{2}<\infty$. As both $n_{t}$ and $n_{r}$ go to infinity, the instantaneous capacity per Rx antenna of the channel is

$$
C \stackrel{p}{\rightarrow}(2 \pi)^{-1} \int_{-\pi}^{\pi} \ln \left[1+\gamma_{0} \lambda(u)\right] d u,
$$

where $\lambda(u)=\lim _{n_{r} \rightarrow \infty} \sum_{n=-n_{r}+1}^{n_{r}-1} R_{k} e^{j n u}, \quad u \in(-\pi ; \pi] \quad$ is the spectrum of $\mathbf{R}_{r}, j=\sqrt{-1}$.

Proof: due to Szego Theorem [6].

Despite the asymptotic nature, (6) and (8) approximate reasonably well the instantaneous capacity of MIMO channels with a moderate number of antennas $\left(n_{t}>n_{r} \geq 2\right)$. To demonstrate this, Fig. 1 shows the capacity of a correlated Rayleigh-fading channel with the Kronecker correlation structure, when both $\mathbf{R}_{t}$ and $\mathbf{R}_{r}$ are given by the exponential correlation model with parameter $r$ (the correlation between two adjacent antennas). The solid line represents the "true" mean capacity obtained by Monte-Carlo (MC) simulation and averaged over $10^{3}$ channel realizations, the error bars illustrate $\pm \sigma$ deviation range of the instantaneous capacity around its mean value, and the doted lines demonstrate the asymptotic approximations in (6) and (8). In general, the gap between (6) and the "true" mean capacity increases with $|r|$. (8) coincides with (6) for small $|r| \leq 0.2$. In contrast to (6), the discrepancy between (8) and the "true" mean capacity decreases with $|r|$, so that the two are practically indistinguishable for $|r| \geq 0.7$. Note that in this case, the deviations of the instantaneous capacity around its mean are relatively small (see Fig. 1), i.e. the latter can be approximated with reasonable accuracy by the mean capacity, which, in turn, is well approximated by the integral in (8).

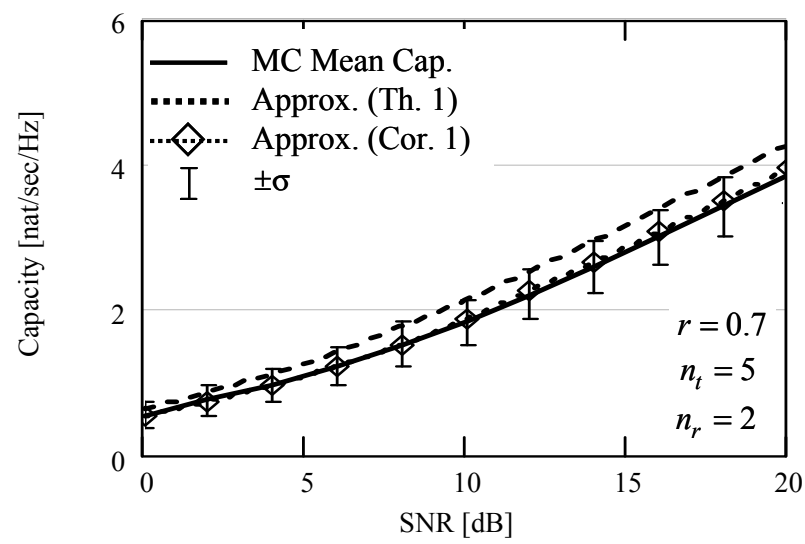

Fig. 1 Capacity per Rx antenna of correlated Rayleigh-fading channel.

Maximum Asymptotic Capacity: Using Hadamard inequality, $0 \leq \operatorname{det}(\mathbf{R}) \leq 1$ for any $n \times n$ correlation matrix $\mathbf{R}$ normalized such that $\operatorname{tr} \mathbf{R}=n$. Thus, it is straightforward to

\footnotetext{
${ }^{2}$ Toeplitz correlation matrix physically corresponds to uniform antenna array geometry, when correlation depends on the spacing between elements only, but not on their positions.
}

show that the asymptotic capacity in (6) is maximal when $\mathbf{R}_{r}=\mathbf{I}$, i.e. the channel is uncorrelated at the $\mathrm{Rx}$ end. In this case the spectrum of $\mathbf{R}_{r}$ is

$$
\lambda(u)=1, u \in(-\pi ; \pi]
$$

and the corresponding channel capacity per $\mathrm{Rx}$ antenna is $C_{\max }=\ln \left(1+\gamma_{0}\right)$, i.e. in the asymptotic approximation, each additional $\mathrm{Rx}$ antenna increases the total capacity by the amount equivalent to the capacity of an 1x1 AWGN channel. The multipath angular density that achieves the maximum $C_{\max }$ for a broad class of MIMO channels is derived in the following sections.

\section{BEST ANGULAR DENSITY}

Consider a channel where the multipath is randomly distributed on the $X Y$ plane. Let $R(x)$ denote the spatial correlation between two antennas at spacing $x$. The multipath wave-number spectrum and the spatial correlation function are related by the Fourier Transform

$$
f\left(k_{x}\right)=(2 \pi)^{-1} \int_{-\infty}^{\infty} R(x) e^{-j k_{x} \cdot x} d x,
$$

where $k_{x}$ is $x$ component of the wave-vector $\mathbf{k} . f\left(k_{x}\right)$ represents the spectral density of the multipath along $x$ axis and is often referred as the probability density function (PDF) of $k_{x}$ due to the following properties: (i) $f\left(k_{x}\right)$ is real and non-negative since $R(x)$ is positive semi-definite, (ii) under normalization $R(0)=1, \int^{\infty} f\left(k_{x}\right) d k_{x}=R(0)=1$.

Consider a receiving ULA of isotropic antennas located along $x$ axis. Let the correlation between antennas be given by a Toeplitz matrix $\mathbf{R}_{r}$, such that $R_{n}=R(d \cdot n), n \in \mathrm{Z}$ (integer numbers), where $R_{n}$ is the matrix-generating vector of $\mathbf{R}_{r}$, and $d$ is the adjacent antenna spacing. From the geometry of the problem, the link between $k_{x}$ and the angle of arrival $\theta$ of a multipath component is $\psi=k_{x} d=2 \pi d \cos \theta,-\pi<\theta \leq \pi$, where $\psi$ represents the phase difference between two adjacent antennas, $\theta$ is measured from the array axis, and $d$ is in wavelengths. Let $f_{\psi}(\psi)$ be the PDF of $\psi$, and $f_{\theta}(\theta)$ be the PDF of $\theta$ or multipath angular density. It is straightforward to show, using the link between the spectra of continuous and sampled signals (via the sampling theorem), that there is a direct relationship between the spectrum $\lambda(u)$ of $\mathbf{R}_{r}$ (see (8)) and $f_{\psi}(\psi)$ as indicated below

$$
\lambda(u)=2 \pi \sum_{n=-\infty}^{\infty} f_{\psi}(u-2 \pi n), u \in(-\pi ; \pi],
$$

where $u$ is the normalized spatial frequency. Combining (9) and (11) one obtains the condition to achieve the maximal capacity $C_{\max } ; C \rightarrow C_{\max }$ if

$$
2 \pi \sum_{n=-\infty}^{\infty} f_{\psi}(\psi-2 \pi n)=1, \psi \in(-\pi ; \pi]
$$

from which we obtain the following.

Theorem 2: (i) If $d<1 / 2$, there is no such $f_{\psi}(\psi)$ that $C \stackrel{p}{\rightarrow} C_{\max }$.

(ii) If $d>1 / 2$, there are a number of $f_{\psi}(\psi)$ such that $C \rightarrow C_{\max }$. 
(iii) If $d=1 / 2$, the only $f_{\psi}(\psi)$ that maximizes the asymptotic capacity per $\mathrm{Rx}$ antenna, is the uniform PDF $f_{\psi}(\psi)=(2 \pi)^{-1}, \quad \psi \in(-\pi ; \pi]$. The corresponding multipath angular density is

$$
f_{\theta}(\theta)=1 / 4 \cdot|\sin (\theta)|, \theta \in(-\pi ; \pi],
$$

and the spatial correlation function is $R(x)=\operatorname{sinc}(2 x)$, where $\operatorname{sinc}(x)=\sin (\pi x) /(\pi x)$.

Proof: via analogy with the zero-ISI Nyquist criterion.

The following remarks to Theorem 2 are in order: Statement (i) of Theorem 2 implies that placing antennas closer than half a wavelength is not optimal in terms of the capacity $C$ per $\mathrm{Rx}$ antenna, which is the measure of efficiency of a single antenna. However, this strategy may increase the capacity per aperture length $C_{a p}=C / d$, which characterizes the efficiency of utilizing the space occupied by the whole antenna array. The following example is the case where the capacity per Rx antenna decreases with $d$, but the capacity per aperture increases. Consider a ULA with $d<1 / 2$ when $f_{\psi}(\psi)$ is uniformly distributed over $[-2 \pi d ; 2 \pi d]$. From Corollary 1 and using (11) for $0<d<1 / 2$,

$$
\begin{gathered}
C \stackrel{p}{\rightarrow}(2 \pi)^{-1} \int_{-2 \pi d}^{2 \pi d} \ln \left[1+2 \pi \gamma_{0} f_{\psi}(u)\right] d u=2 d \ln \left[1+\gamma_{0} /(2 d)\right] \\
C_{a p}=C / d \stackrel{p}{\rightarrow} 2 \ln \left[1+\gamma_{0} /(2 d)\right]
\end{gathered}
$$

i.e. asymptotically $C$ decreases as $d$ decreases, but $C_{a p}$ increases.

An example $f_{\psi}(\psi)$, which satisfies the condition in (12) for $1 / 2 \leq d \leq 1$ and therefore maximizes the asymptotic capacity, is $f_{\psi}(\psi)=S_{R C}(\psi)$, where $S_{R C}(\psi)$ is the raised cosine spectrum with the bandwidth $B=2 \pi d$ and the roll-off factor $\beta=2 d-1$. The best angular density in this case is given by

$$
f_{\theta}(\theta)=2 \pi d \cdot S_{R C}(2 \pi d \cos \theta) \cdot \sin (\theta), \theta \in[0 ; \pi]
$$

(due to the problem's symmetry, we consider the half-space $\theta \in[0 ; \pi]$ only). For $d=1,(15)$ reduces to

$$
f_{\theta}(\theta)=\cos ^{2}(\pi / 2 \cos \theta) \cdot \sin (\theta)
$$

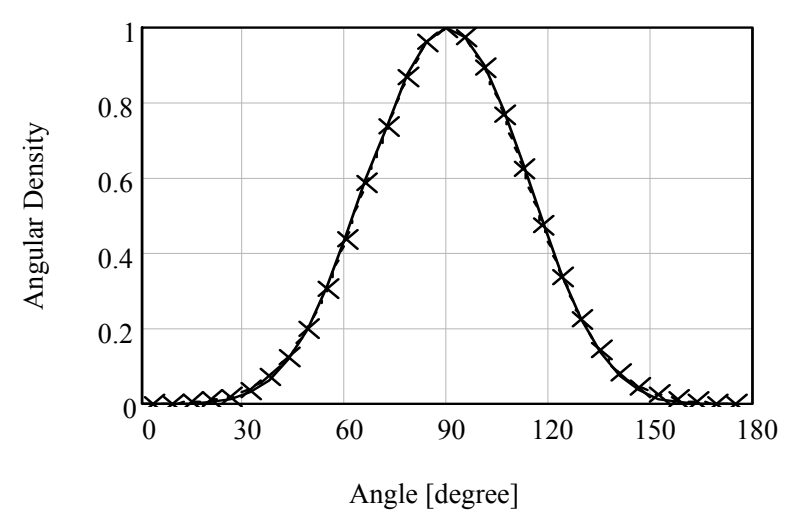

Fig. 2 Comparison between the best multipath angular density (16) (solid line) and its Gaussian approximation (x's line).
We notice that (16) is accurately approximated by the Gaussian distribution $N(\mu, \sigma)$ with the peak is at the array broadside $\mu=90^{\circ}$, and the multipath spread around the peak is $\sigma=(2 \pi)^{-1 / 2} \approx 23^{\circ}$. As Fig. 2 shows, the Gaussian distribution and the one in (16) are virtually indistinguishable, from which we conclude that, in terms of the capacity, the Gaussian angular density (adopted in IEEE 802.11n [4]) is very close to the optimum one for $d=1$.

When $d>1$, it is generally difficult to find what angular density is the best, even though the existence of such density is implied by statement (ii) of Theorem 2 . However, as $d \rightarrow \infty$, the following corollary gives a simple necessary condition that applies to $f_{\theta}(\theta)$ and indirectly verifies (12).

Corollary 2.1: For any multipath angular density that satisfies $f_{\theta}(\pi / 2)<\infty$, i.e. there is no specular component at the broadside direction of the ULA, (12) always holds true as $d \rightarrow \infty$, and therefore $C \stackrel{p}{\rightarrow} C_{\max }$.

Proof: based on the property that $\lim _{x \rightarrow \infty} R(x)=0$, $f_{\theta}(\pi / 2)<\infty$.

Consider a special case when Corollary 2.1 is not satisfied. Assume that specular multipath arrive at only two equiprobable angles $\pm \pi / 2$, i.e.

$$
f_{\theta}(\theta)=[\delta(\theta-\pi / 2)+\delta(\theta+\pi / 2)] / 2,
$$

where $\delta(x)$ is the Dirac's delta function. Then it is straightforward to show that in this case $\lambda(u)=2 \pi \delta(u)$, and therefore from Corollary 1

$$
C \stackrel{p}{\rightarrow}(2 \pi)^{-1} \lim _{\varepsilon \rightarrow 0^{+}} \int_{-\varepsilon}^{\varepsilon} \ln \left[1+\pi \gamma_{0} / \varepsilon\right] d u=0,
$$

i.e. $C$ does not converge to $C_{\max }$ regardless of $d$. Moreover, the contribution of each single antenna to the total capacity, in this case, approaches zero as $n_{r} \rightarrow \infty$.

Statement (iii) of Theorem 2 indicates that when $d=1 / 2$, the only multipath angular density $f_{\theta}(\theta)$ that maximizes the asymptotic capacity is non-uniform, i.e. for asymptotically large ULA, the Clarke's (Jakes's) model, where $f_{\theta}(\theta)$ is assumed to be uniform over $(-\pi, \pi]$, does not represent the best case scenario. Since, in some cases, the asymptotic capacity approximates well the exact one (see Fig. 1), the same conclusion can be also made for ULA with a moderate number of antennas. Note that following the discussion in Section II, this conclusion holds for a broad class of MIMO channels without a specific correlation structure or not necessarily Rayleigh-fading.

\section{REFERENCES}

[1] A. M. Sayeed and V. Raghavan, "Maximizing MIMO Capacity in Sparse Multipath with Reconfigurable Antenna Arrays," IEEE Journal of Selected Topics in Signal Processing, vol.1, no.1, pp.156-166, June 2007.

[2] T. S. Pollock et al, "Antenna Saturation Effects on Dense Array MIMO Capacity”, ICASSP 2003, pp. IV-361-4, Apr. 2003.

[3] D. Chizhik et al, "Effect of Antenna Separation on the Capacity of BLAST in Correlated Channels," IEEE Communications Letters, vol.4, no.11, pp. 337-339, Nov 2000.

[4] V. Erceg et al, "TGn Channel Models", Proposal for IEEE P802.11 Wireless LANs, Doc. IEEE 802.11-03/940r4, May 2004.

[5] A. M. Tulino, et al, "Impact of Antenna Correlation on the Capacity of Multiantenna Channels," IEEE Trans. on Information Theory, vol.51, no.7, pp. 2491-2509, July 2005.

[6] R. M. Gray, "Toeplitz and Circulant Matrices: A Review", Foundations and Trends in Commun. and Inform. Theory, vol. 2, no 3, pp. 155-239, Aug. 2002. 